\title{
Auxiliary or orthotopic liver transplantation for acute fatty liver of pregnancy: case series and review of the literature
}

\author{
J Ringers, ${ }^{a}$ KWM Bloemenkamp, ${ }^{\text {b }}$ N Francisco, ${ }^{c}$ JJ Blok, ${ }^{a}$ MS Arbous, ${ }^{d}$ B van Hoek ${ }^{c}$ \\ a Department of Transplant Surgery, Leiden University Medical Centre, Leiden, the Netherlands ${ }^{\mathrm{b}}$ Department of Obstetrics and Gynaecology, \\ Leiden University Medical Centre, Leiden, the Netherlands ${ }^{c}$ Department of Gastroenterology and Hepatology, Leiden University Medical \\ Centre, Leiden, the Netherlands ${ }^{\mathrm{d}}$ Department of Intensive Care, Leiden University Medical Centre, Leiden, the Netherlands \\ Correspondence: Prof Dr B van Hoek, Department of Gastroenterology and Hepatology, C4-P, Leiden University Medical Centre, PO \\ Box 9600, 2300 RC Leiden, the Netherlands. Email b.van_hoek@lumc.nl
}

Accepted 12 August 2015. Published online 9 October 2015.

Please cite this paper as: Ringers J, Bloemenkamp KWM, Francisco N, Blok JJ, Arbous MS, van Hoek B. Auxiliary or orthotopic liver transplantation for acute fatty liver of pregnancy: case series and review of the literature. BJOG 2016;123:1394-1398.

\section{Case series}

The objective of this study was to present all cases of liver transplantation (LT) for acute fatty liver of pregnancy (AFLP) from 1979 through 2012 in the Netherlands and to review the literature.

\section{Orthotopic liver transplantation}

The first case was a 30-year-old woman with a history of two uneventful pregnancies and deliveries. In the first trimester of her third pregnancy she had suffered temporarily from hyperemesis gravidarum. In 2002, in the 25th week of pregnancy, she presented elsewhere with jaundice, itching, upper abdominal pain, nausea, vomiting and fatigue. She then rapidly developed encephalopathy and disturbed clotting, and had a spontaneous vaginal delivery of a boy at 25 weeks with postpartum haemorrhage. The baby died the first day. Because bilirubin, International Normalised Ratio (INR) and encephalopathy further deteriorated, 4 days after delivery she was admitted to the intensive care unit of the Leiden University Medical Centre (LUMC). There was jaundice and severe hepatic encephalopathy with restlessness, and coma developed within 1 day (Opolon-Conn stage 4, EMV score 3). No signs of chronic liver disease and no Kaiser-Fleisher rings were present. An underlying autoimmune disorder, viral or metabolic causes such as Wilson disease were excluded. Abdominal Doppler ultrasound revealed hepatic steatosis but was otherwise normal. A liver biopsy showed some hepatocyte necrosis and evident steatosis, $80 \%$ microvesicular and 20\% macrovascular, compatible with a diagnosis of AFLP. Laboratory and clinical findings are shown in Table 1.
The patient fulfilled King's College Hospital $(\mathrm{KCH})$ criteria for LT. ${ }^{1}$ She was placed on the high urgency waiting list for LT and was supported by continuous venovenous haemofiltration $(\mathrm{CVVH})$ with molecular absorbent recirculating system (MARS), intravenous acetylcysteine and systemic cooling $\left(35^{\circ} \mathrm{C}\right)$ until orthotopic liver transplantation (OLT). ${ }^{2}$ The next day she underwent OLT. Postoperatively, she developed renal insufficiency but CVVH was not necessary because renal function rapidly improved. She was discharged home on day 22 after OLT without further complications and with tacrolimus and mycophenolate mofetil as immunosuppressants. No long-chain hydroxacyl CoA dehydrogenase (LCHAD) mutations were detected. ${ }^{3}$ She has remained well more than 10 years after OLT, without further pregnancies.

\section{Auxiliary liver transplantation}

The other woman was 35 years of age, with a history of two spontaneous miscarriages. During her third pregnancy she presented in 2002 at another hospital at 37 weeks gestation with fatigue, drowsiness and nausea, haemodynamic instability and intra-uterine fetal death. Delivery was induced. Six days postpartum she was referred to LUMC with progressive liver failure with disturbed clotting, renal insufficiency, hypoglycaemia and haemodynamic instability. Within a day she developed hepatic coma (Opolon-Conn stage 4, EMV score 3) and brain oedema as evidenced by transcranial Doppler examination. Laboratory and clinical findings are indicated in Table 1 . She did not fully comply with $\mathrm{KCH}$ criteria, but serum lactate was $8.9 \mathrm{mmol} / \mathrm{l}$. Immediate treatment was started with $\mathrm{CVVH}$ with MARS, intravenous acetylcysteine and systemic cooling $\left(35^{\circ} \mathrm{C}\right)$. 


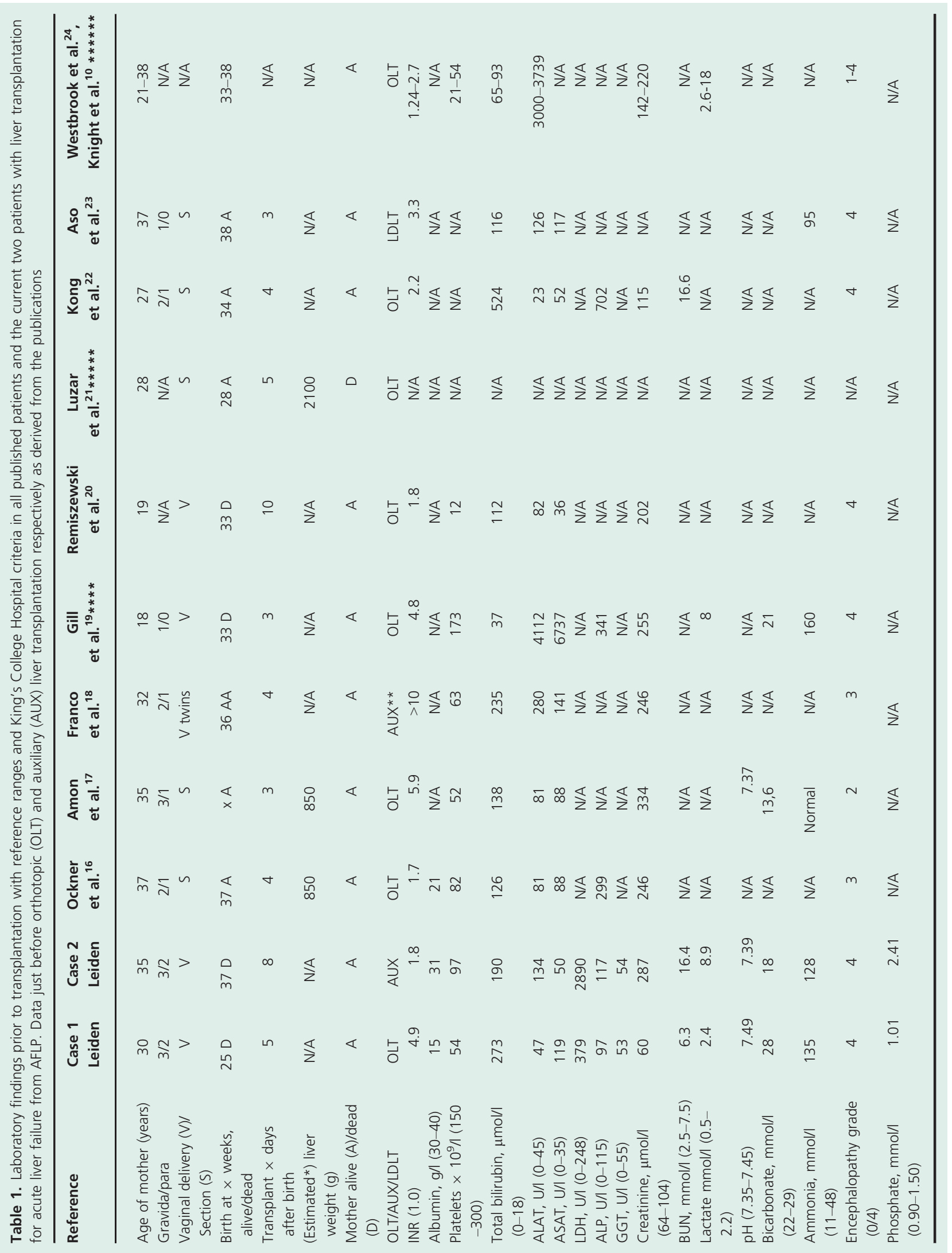




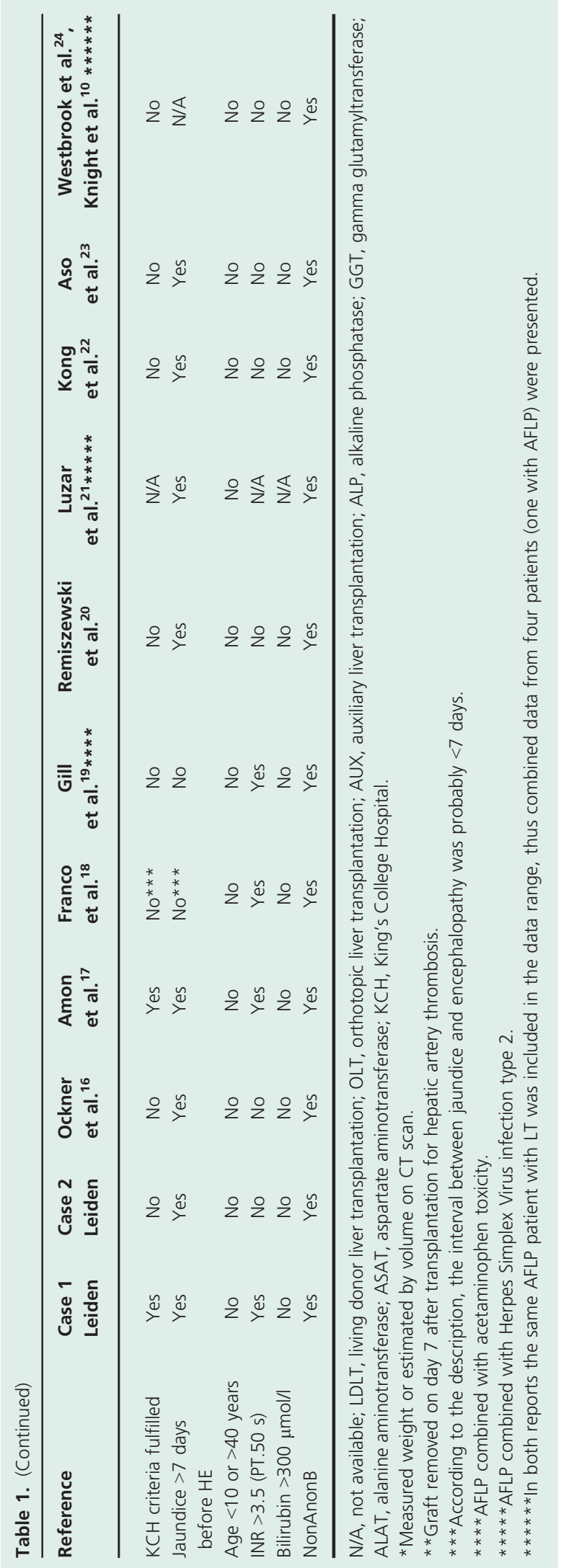

She was placed on the high urgency waiting list for LT. The next day, auxiliary liver transplantation (AUX) was carried out, with a left partial graft (segments 2, 3 and half of 4) in the left upper abdomen and a renoportal anastomosis, leaving the native liver untouched, as decribed elsewhere. ${ }^{4}$ An intra-operative native liver biopsy revealed severe steatosis, mixed micro- and macrovascular (50/50\%), and was compatible with a diagnosis of AFLP. Postoperatively, CVVH was required and critical illness neuropathy developed. This resolved and the patient recovered within 3 weeks. Both livers were regularly assessed with hepatobiliary scintigraphy and computed tomography with volumetry and liver biopsies. Scintigraphy showed sufficient function of the graft and improving function of the native liver over time, and liver biopsies showed a significant reduction of steatosis in the native liver. This led to removal of the auxiliary liver on day 31 after AUX. This liver was re-used as an orthotopic graft in another recipient, as decribed elsewhere. ${ }^{5}$ Tacrolimus and prednisolone were stopped. She went home on day 7 after graft explantation. No LCHAD mutations were found. Now, more than 10 years later, she leads a normal life with normal liver function, without further pregnancies.

\section{Discussion}

Searching the databases from the three Dutch liver transplant centres revealed that in 33 years of liver transplantation in 1979-2012 in the Netherlands, there were two cases - one OLT and one AUX - from 2445 liver transplantations $(<0.1 \%)$ for AFLP. Both had an excellent outcome. After AUX, the graft could be removed and immunosuppression stopped after regeneration of the native liver. The graft was then successfully used for OLT in another recipient.

After obtaining permission to search in the databases of the European Liver Transplant Registry and in the Eurotransplant database, under-reporting and wrong coding were found, preventing further European analysis.

AFLP can occur in the third trimester of pregnancy, with an estimated prevalence of 1:7000-13 000 deliveries. Maternal mortality of AFLP was $80 \%$ before 1980, and $18 \%$ in 1999 , with a fetal mortality rate of $20-60 \%{ }^{6,7}$ This improvement is probably due to many factors, such as improved intensive care and better obstetric care, including earlier termination of pregnancy. ${ }^{2,6-8}$ Patients often present with epigastric pain, nausea and vomiting. Jaundice, hepatic encephalopathy, thrombocytopenia and disturbed clotting can be present, and subcapsular haematomas and liver rupture can occur. The Swansea criteria for diagnosing AFLP were validated in a prospective study. ${ }^{9,10}$ Overlap with HELLP (haemolysis, elevated liver enzymes and low platelet count) syndrome can 
exist. Treatment consists in accelerated delivery with correction of clotting and of hypoglycaemia, and most women recover. Infants receive an adjusted diet, rich in carbohydrates and low-fat, and long-term fasting is avoided. ${ }^{6,7}$ In acute liver failure (ALF), early CVVH with cooling and intravenous antibiotics may reduce the incidence of brain oedema, one of the major causes of death in ALF. $^{2}$ MARS is still used in ALF but has no proven survival benefit. In contrast, plasmapheresis may be beneficial in AFLP, and this may also be true for ALF in general. $^{11,12}$

Between August 2004 and August 2006 in the Netherlands there were 12 cases of severe maternal morbidity from AFLP, representing $0.47 \%$ of the 2552 cases of severe maternal morbidity from a total of 371021 deliveries. $^{13}$ This gives an incidence of AFLP of 3.2 cases per 10000 deliveries (95\% CI 1.8-5.7). In this period no women died due to AFLP. If we extrapolate the 12 cases in these 2 years to 23 years, then this represents $23 * 12 / 2=138$ cases of severe maternal morbidity from AFLP. In the 23year period 1983-2006, the maternal mortality rate from AFLP was 0.13 per 100000 live births (95\% CI 0.050.29 ), as reported in a retrospective study, while maternal death from AFLP occurred in six women (one in 1995, five in 2000-2001) from 538 cases (1\%) of maternal death. ${ }^{14}$ In four of these six cases, liver failure was mentioned as a cause of death. In the other two cases the deaths were likely related to the AFLP, but with diffuse intravascular coagulation (DIC) and with cardiac arrest as the cause. Two LTs for AFLP were performed in the Netherlands in 33 years; in 23 years this would be $2 * 23 /$ $33=1.4$. Assuming that the two women we transplanted would otherwise have died, and adding the four cases of maternal death due to liver failure from AFLP to the above calculated incidence of LT of 1.4 in 23 years assuming these patients could have been saved by LT LT would have been required in $4+1.4=5.4$ of 138 (3.9\%) patients with severe morbidity from AFLP. Obviously, this is only a rough estimation: data do not span the same years, and it was assumed that all women who died from liver failure due to AFLP would benefit from LT, which may not be the case. Some may have been too sick to undergo LT. Furthermore, sepsis with jaundice and DIC can mimic ALF, and such patients should not be transplanted. A recent prospective study from the UK found severe morbidity from AFLP of 31 per $10^{6}$ births. $^{15}$ This is in very good agreement with the severe AFLP incidence found in the Netherlands of 32 per $10^{6}$ births. $^{13}$ In the UK, 0.7 deaths per $10^{6}$ births were due to AFLP, with a survival-to-death ratio for AFLP of 70:1. ${ }^{15}$ Thus in all the British cases with AFLP, including those less severe, a maximum of $1 / 71$ (1.4\%) women may need LT. This indeed is close to the case fatality rate for AFLP in the
UKOSS study of $1.8 \%$ (95\% CI $0-9.4 \%) .{ }^{10}$ In the Netherlands we included only cases of severe AFLP in the calculation above, which explains the difference between 3.9\% and $1.4 \%$, but the figures are within the same range.

In the UKOSS study, infant perinatal mortality in mothers with AFLP was around $10 \% .^{10}$

In total, 11 women, including the two Dutch cases, with LT for AFLP have been published, as summarised in Table $1 .^{10,16-24}$ Ten of the 11 survived. This may partially be publication bias, as it is possible that non-survivors were not published. Nevertheless, these data show that LT can be lifesaving in very severe AFLP without recovery after termination of pregnancy. Most transplants were performed within a week after delivery.

The decision to perform LT is a difficult one, especially as the $\mathrm{KCH}$ criteria for LT in ALF apparently do not fully apply to AFLP. This is due to aggressive correction of coagulopathy for bleeding complications, resulting in a lower INR, where age is below 40, and bilirubin often below $300 \mu \mathrm{mol} / \mathrm{l}$, and the interval between development of jaundice and encephalopathy is usually shorter than a week. ${ }^{24}$ Our data from the transplanted patients confirm this. Data from $\mathrm{KCH}$ also showed that in women with severe maternal morbidity requiring intensive care admission (almost all with AFLP or hypertensive disease in pregnancy), the presence of encephalopathy and lactic acidosis seem better than the $\mathrm{KCH}$ criteria to distinguish women requiring LT from those who will survive without $\mathrm{LT}^{24}$ Indeed, all women transplanted for AFLP shown in Table 1 had hepatic encephalopathy and in the few with measured serum lactate and ammonia, these values were elevated. This needs to be validated further. Especially as most patients with AFLP recover after delivery, better guidelines for indicating LT in AFLP are required.

Although OLT is the standard, an advantage of AUX for ALF is that in the majority of surviving patients the native liver regenerates, and withdrawal of immunosuppression is possible within a year, with removal of the graft or graft atrophy after deliberate chronic rejection. ${ }^{25,26}$ In haemodynamically unstable women with 'toxic liver syndrome' due to cytokine release from liver necrosis, OLT may be preferred. As severe necrosis is unusual in AFLP, AFLP without improvement after delivery and with hepatic encephalopathy and elevated serum lactate and ammonia may be an ideal indication for AUX in haemodynamically stable women, but this needs further confirmation. AUX is a more complex operation than OLT with some more primary malfunction and vascular complications, but it may allow timely intervention and withdrawal of immunosuppression.

\section{Disclosure of interests}

Full disclosure of interests available to view online as supporting information. 


\section{Contribution to authorship}

All individuals that qualified for authorship have been included and all those included qualify for authorship. JR, $\mathrm{KWMB}, \mathrm{MSA}$ and $\mathrm{BvH}$ contributed to conception and design, interpreted the data, NF and JJB acquired the data, $\mathrm{BvH}$ and JR drafted the article, all authors revised the article critically for intellectual content and approved the final version to be published, and agreed to be accountable for all aspects of the work, ensuring that questions related to the accuracy or integrity of any part of the work were appropriately investigated and resolved.

\section{Details of ethics approval}

Retrospective studies with anonymised reporting are by law exempt from approval by the institutional review board. Both patients gave informed consent for this report, which conformed to the ethical guidelines of the 1975 Declaration of Helsinki.

\section{Funding}

None.

\section{Acknowledgements}

We thank Prof. Dr. R. J. Porte, surgeon of the University Medical Center Groningen, the Netherlands and Prof. Dr. H. J. Metselaar, hepatologist of the Erasmus University Medical Centre in Rotterdam, the Netherlands, Eurotransplant and the ELITA-ELTR for allowing us to search their liver transplant databases.

\section{References}

1 O'Grady JG, Alexander GJ, Hayllar KM, Williams R. Early indicators of prognosis in fulminant hepatic failure. Gastroenterology 1989:97:439-45.

2 Bernal W, Wendon J. Acute liver failure. $N$ Engl J Med 2013;369:2525-34

3 Ibdah JA, Bennett MJ, Rinaldo P, Zhao Y, Gibson B, Sims HF, et al. A fetal fatty-acid oxidation disorder as a cause of liver disease in pregnant women. N Engl J Med 1999;340:1723-31.

4 Ringers J, Baranski AG, Dubbeld J, Sarton E, Veenendaal RA, Schaapherder AF, et al. A novel technique for auxiliary partial liver transplantation with reno-portal anastomosis and avoidance of the hepatoduodenal ligament. Am J Transplant 2006;6:2802-8.

5 Ringers J, Dubbeld J, Baranski AG, Coenraad M, Sarton E, Schaapherder $A F$, et al. Reuse of auxiliary liver grafts in second recipients with chronic liver disease. Am J Transplant 2007;7:2615-8.

6 Hay JE. Liver disease in pregnancy. Hepatology 2008;47:1067-76.

7 Pan C, Perumalswami PV. Pregnancy-related liver diseases. Clin Liver Dis 2011;15:199-208.

8 Bernal W1, Hyyrylainen A, Gera A, Audimoolam VK, McPhail MJ, Auzinger $G$, et al. Lessons from look-back in acute liver failure? A single centre experience of 3300 patients J Hepatol 2013;59:74-80.

9 Ch'ng CL, Morgan M, Hainsworth I, Kingham JG. Prospective study of liver dysfunction in pregnancy in Southwest Wales. Gut 2002;51:876-80
10 Knight M, Nelson-Piercy C, Kurinczuk JJ, Spark P, Brocklehurst P. A prospective national study of acute fatty liver of pregnancy in the UK. Gut 2008;57:951-6.

11 Martin JN Jr, Briery CM, Rose CH, Owens MT, Bofill JA, Files JC. Postpartum plasma exchange as adjunctive therapy for severe acute fatty liver of pregnancy. J Clin Apher 2008;23:138-43.

12 Larsen FS, Schmidt LE, Bernsmeier C, Rasmussen A, Isoniemi $H$, Petel VC, et al. High-volume plasma exchange in patients with acute liver failure: an open randomised controlled trial. J Hepatol 2015. doi: 10.1016/j/jhep.2015.08.018.

13 Zwart JJ, Richters JM, Ory F, de Vries JI, Bloemenkamp KW, van Roosmalen J. Severe maternal morbidity during pregnancy, delivery and puerperium in the Netherlands: a nationwide population-based study of 371,000 pregnancies. BJOG 2008;115: 842-50.

14 Dekker RR, Schutte JM, Stekelenburg J, Zwart JJ, van Roosmalen J. Maternal mortality and severe maternal morbidity from acute fatty liver of pregnancy in the Netherlands. Eur J Obstet Gynecol Reprod Biol 2011;157:27-31

15 Kayem G, Kurinczuk J, Lewis G, Golightly S, Brocklehurst P, Knight $M$. Risk factors for progression from severe maternal morbidity to death: a national cohort study. PLOS ONE 2011;6:e29077.

16 Ockner SA, Brunt EM, Cohn SM, Krul ES, Hanto DW, Peters MG Fulminant hepatic failure caused by acute fatty liver of pregnancy treated by orthotopic liver transplantation. Hepatology 1990;11:59-64.

17 Amon E, Allen SR, Petrie RH, Belew JE. Acute fatty liver of pregnancy associated with preeclampsia: management of hepatic failure with postpartum liver transplantation. Am J Perinato/ 1991;8:278-9.

18 Franco J, Newcomer J, Adams M, Saeian K. Auxiliary liver transplant in acute fatty liver of pregnancy. Obstet Gynecol 2000; 95:1042.

19 Gill EJ, Contos MJ, Peng TC. Acute fatty liver of pregnancy and acetaminophen toxicity leading to liver failure and postpartum liver transplantation. A case report. J Reprod Med 2002;47:584-6.

20 Remiszewski P, Pawlak J, Skwarek A, Grzelak I, Patkowski W, Grodzicki $\mathrm{M}$, et al. Orthotopic liver transplantation for acute liver failure resulting from 'acute fatty liver of pregnancy'. Ann Transplant 2003;8:8-11.

21 Luzar B, Ferlan-Marolt V, Poljak M, Sojar V, Stanisavljevic D, Bukovac $T$, et al. Acute fatty liver of pregnancy-an underlying condition for herpes simplex type 2 fulminant hepatitis necessitating liver transplantation. Z Gastroenterol 2005;43:451-4.

22 Kong XF, Zhang XX, Yu YY, Shi Q, La DD, Zhu-Ge CD, et al. No mutation was found in the alpha-subunit of the mitochondrial trifunctional protein in one patient with severe acute fatty liver of pregnancy and her relatives. J Gastroenterol Hepatol 2007;22:2107-11.

23 Aso K, Hojo S, Yumoto Y, Fukushima K, Miyazaki M, Kotoh K, et al. Three cases of acute fatty liver of pregnancy: postpartum clinical course depends on interval between onset of symptoms and termination of pregnancy. J Matern Fetal Neonatal Med 2010;23:1047-9.

24 Westbrook RH, Yeoman AD, Joshi D, Heaton ND, Quaglia A, O'Grady JG, et al. Outcomes of severe pregnancy-related liver disease: refining the role of transplantation. Am J Transplant 2010;10:2520-6.

25 van Hoek B, de Boer J, Boudjema K, Williams R, Corsmit O, Terpstra OT. Auxiliary versus orthotopic liver transplantation for acute liver failure. EURALT Study Group. European Auxiliary Liver Transplant Registry. J Hepatol 1999;30:699-705.

26 Quaglia A, Portmann BC, Knisely AS, Srinivasan P, Muiesan P, Wendon J, et al. Auxiliary transplantation for acute liver failure: histopathological study of native liver regeneration. Liver Transp/ 2008; 14:1437-48. 